\title{
2. A Determination of Meteor Mass Distribution From Meteor Echoes
}

\author{
G. For'ri* \\ Smithsonian Astrophysical Observatory \\ Cambridge, Massachusetts
}

\begin{abstract}
Meteor counts from the observations of the Harvard-Smithsonian Radio Meteor Project at four magnitudes in the range of about +9 to +15 in the visual scale were used to determine the exponent of the mass-distribution law under certain assumptions. Since for these data no range measures were available, and since the pattern of the transmitting/ receiving antennas was very broad, the same range and radiant distribution were assumed for all counts within the same half-hour during which individual counts at different magnitudes were obtained. These echo counts, covering in five successive days $24 \mathrm{hr}$ with overlapping periods, yielded for the exponent a value of $1.95 \pm 0.02$, close to other determinations. Individual values, however, presented a considerable scatter, probably reflecting changes in radiant and mass distribution over the year. From the same data, it appeared that the mass exponent changed during the day, yielding generally a higher value for the evening hours.
\end{abstract}

The Number of METeoroids entering the 1 Earth's atmosphere increases as the numerical value of the magnitude increases. If the number $N_{m}$ of meteoroids having mass $m$ and larger can be expressed as

$$
N_{m} \propto m^{-8+1}
$$

then, assuming a linear dependence between the mass and the electron line density $q$ produced by a meteoroid, the distribution of meteor trails with line density $q$ and larger will be

$$
N_{q} \propto q^{-8+1}
$$

If the number $N_{q}$ is known at different limiting values of $q$, a determination of the mass exponent $s \dagger$ can be attempted. The mass exponent $s$ has been computed from meteor data

* Present address: Osservatorio Astrofisico Di Arcetri, Firenze, Italy.

$\dagger s$ has also been termed the differential mass index. at different magnitudes; its value ranges between 2.5 and 2.2. Hawkins and Upton (1958) find a value 2.34 for $s$ over a magnitude interval -1 to +4 ; Šimek and McIntosh (1968), 2.35 between magnitudes +5 and +10 ; Kaiser (1961), 2.17 between magnitudes +8 and +11 ; and Öpik (1958), $\sim 2.2$ between magnitudes +2 and +5 . The recent results of McIntosh and Simek (1969) do not change the conclusions of their earlier paper.

Meteor counts at four different limiting magnitudes, from approximately +9 to +15 absolute visual magnitude, were recorded during normal operations of the Harvard-Smithsonian Radio Meteor Project, Havana, Ill., lat. $40^{\circ} 13^{\prime} \mathrm{N}$, long. $90^{\circ} 01^{\prime} \mathrm{W}$, and $s$ was derived from these observations. The radar operates at a wavelength of $7.331 \mathrm{~m}$, with a peak power of about $3 \mathrm{MW}$ and antenna gains near $20 \mathrm{db}$ over an isotropic radiator. 
The electron line density is known to be dependent on the meteor's velocity and zenith angle as well as on its mass, and the observed velocity and radiant distribution vary over the day. In consequence, diurnal average values of $s$ are more meaningful here than values at particular times of day. It has not been possible to correct the data for observational height limitations, which must have some effect. The diffusion ceiling and recombination floor on heights (Southworth, paper no. 3 this volume) will eliminate some relatively small and large line densities, respectively. Nonethcless, since we unexpectedly observed many large and small line densities compared with intermediate densities, diffusion and recombination appear to be unimportant to an overall determination of $s$.

\section{ECHO COUNTS}

The observed meteor rates are automatically recorded by the radar equipment, which counts the meteor echoes received in given intervals of time, down to four different limiting receiver sensitivities covering the useful dynamic range of the system. The magnitude range is about $+9<M<+15$ in the radar scale, which corresponds roughly with the visual absolute magnitude scale.

Counts are obtained every half-hour, with each receiver level sampled at least once. Counts for the faintest meteors, detected down to the receiver noise, are increased by spurious echoes, even though the equipment was specifically designed to kcep these events to a minimum. Spurious counts are probably recorded at the lowest level of receiver sensitivity as well, owing to airplane echoes from a nearby airport. Both limitations must be considered in the analysis of the data. During the recording, the transmitted peak power is monitored, as well as the receiver sensitivities at each level. Observations are usually carried out on five consecutive days, each with an average of about $10 \mathrm{hr}$ of continuous recording. The periods of recording are shifted to cover a full $24 \mathrm{hr}$ with overlapping observations.

From these counts, it is possible to derive a diurnal variation of rate that represents the observations of a whole week. The sums of the counts of each half-hour at each level of sensitivity are normalized to this observed diurnalrate curve to allow for the fact that these data are obtained on different days and over different periods of the day, during which the meteor rates can change considerably.

\section{ANALYSIS AND RESULTS}

Rates are discussed here in terms of a convenient parameter

$$
L=10^{10} \sqrt{P_{R} / P_{T}}
$$

where $P_{T}$ is the transmitter power and $P_{R}$ is the limiting receiver sensitivity; both varied slightly from time to time. With a mean antenna power gain of 110 and a mean slant range of $135 \mathrm{~km}$, the mean limiting electron line density is

$$
q=4.5 \times 10^{9} \mathrm{~L}
$$

Denoting by $N_{L}$ the hourly number of meteor echoes exceeding a given $L$, a least-squares fit of $\log _{10} N_{L}$ versus $\log _{10} L$ has been made for the data covering the period October 1968 to November 1969 .

A linear fit was made for each of the 24 weeks of observations and for selected groups of hours from the reconstructed daily rates. Proper weights were introduced in the least-squares solutions to take into account false events and statistical fluctuations in $N_{L}$. The variation of the exponent $s$ with time is plotted in figure 1 , where the dotted line indicates the results from the mean rate over $24 \mathrm{hr}$, and the solid and the dashed lines show $s$ from the mean of $4 \mathrm{hr}$ in the morning ( $5^{\mathrm{h}}$ to $\left.8^{\mathrm{h}}\right)$ and in the evening $\left(17^{\mathrm{h}}\right.$ to $20^{\mathrm{h}}$ ), respectively. The slope $1-\dot{s}$, derived from a fit combining all the data, is shown in figure 2, where the mean $N_{L}$ over $24 \mathrm{hr}$ is plotted versus $L$. The values of $s$ for the different mean rates, together with their errors, are given in table 1.

The morning observations include a relatively large number of retrograde orbits, and the evening hours, of direct low-eccentricity orbits. The large counts from the ecliptic streams of moderate eccentricity are included only in the 24-hr averages. Since there are still other diurnal variations, it is difficult to interpret the apparent diurnal changes in $s$. 


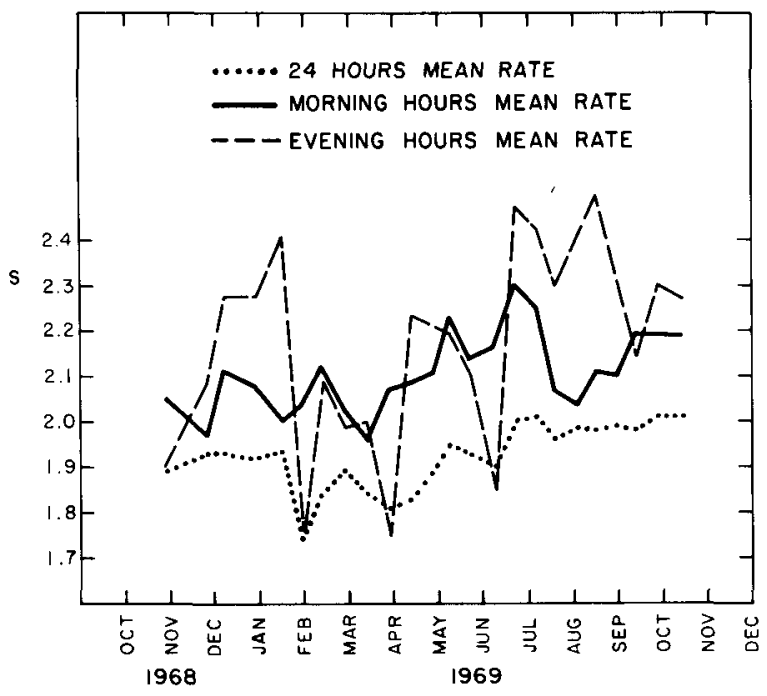

FIGURE 1.-Exponent $s$ as a function of the date.

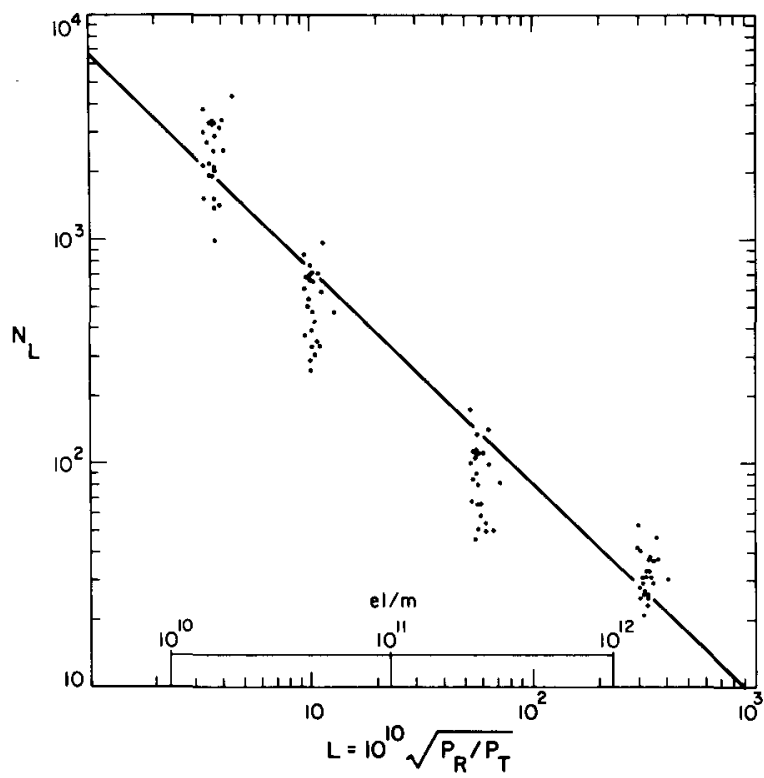

Figure 2.-Counts $N_{L}$ plotted against $L$. Straight line: $s$ from linear fit.

A mean daily variation of meteor rates, plotted in figure 3, was deduced from all the data for each of the four limiting magnitudes. Similar theoretical curves were obtained by Elford and Hawkins (1964) from the study of the orbits of sporadic meteors and from the geometry of the radar system.

The curves of figure 4 , where the mean rate for each week of observations is plotted versus
TABLE 1.-Exponent $\mathrm{s}$ and its Error for Different Mean Rates

\begin{tabular}{|c|c|c|c|}
\hline \multirow{2}{*}{$\begin{array}{c}\text { Magnitude } \\
\text { interval }\end{array}$} & \multirow{2}{*}{ Mean rate } & \multicolumn{2}{|c|}{$\begin{array}{l}\text { First-order } \\
\text { polynomial }\end{array}$} \\
\hline & & $s$ & $\begin{array}{c}\text { Standard } \\
\text { error }\end{array}$ \\
\hline \multirow{4}{*}{$+9<M<+15$} & (24 hours & 1.95 & 0.02 \\
\hline & Morning hours & 2.14 & 0.05 \\
\hline & Evening hours & 2.26 & 0.10 \\
\hline & 24 hours & 2.19 & 0.04 \\
\hline \multirow[t]{3}{*}{$+11<M<+15$} & Morning hours & 2.23 & 0.07 \\
\hline & Evening hours & 2.49 & 0.14 \\
\hline & 24 hours & 1.82 & 0.03 \\
\hline \multirow[t]{2}{*}{$+9<M<+13$} & Morning hours & 1.96 & 0.05 \\
\hline & Evening hours & 1.85 & 0.08 \\
\hline
\end{tabular}

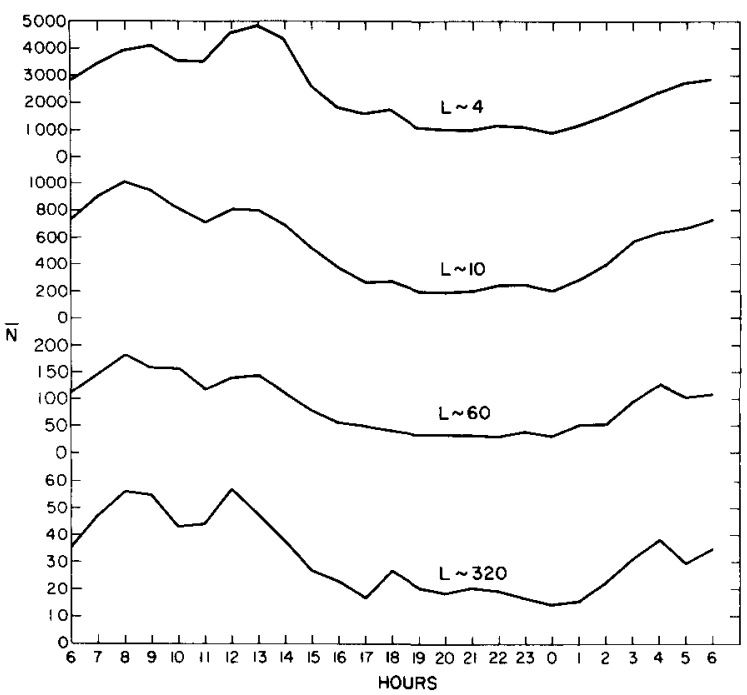

Figdre 3.-Diurnal variation in the echo rates from all the data.

the time of the year, are in good agreement with the distribution of sporadic meteors over the year as found by Kresáková and Kresák (1955) from telescopic observations and by Weiss (1957) from radar echoes. This accordance among different surveys seems to indicate that the distribution of sporadic meteors does not change significantly with the years.

\section{CONCLUSIONS}

The observed rate of radio meteors depends on many factors, the most important being the 


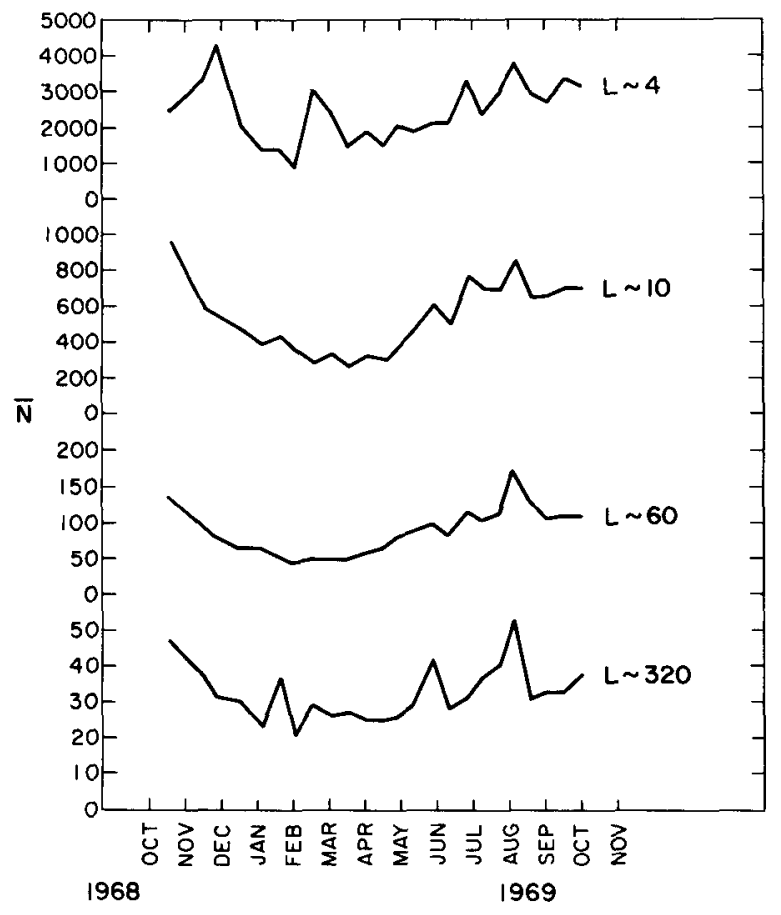

Figure 4.-Mean rates as a function of time of the year.

equipment parameters, the ionizing process, and the radiant distribution. To calculate the mass exponent $s$ from the echo counts obtained in Havana, it was necessary to make some assumptions and assign certain values to unknown constants. First, since no range measures are available for these counts, the same range distribution was assumed at each limiting magnitude; second, with the antenna pattern very broad and the side lobes conspicuous, the same radiant distribution was assumed for all the counts of each half-hour of recording. The true count of meteor echoes exceeding a certain minimum electron line density is undoubtedly different from the observed count because of the lack of knowledge of range and antenna gain for each returned echo. Once these parameters are assumed, the exponent $s$ can be calculated with some confidence.

The value found for $s$ is $1.95 \pm 0.02$; it is lower than the determinations cited above but close to the values of Kaiser (1961) and Opik (1958). When the exponent is determined over a different magnitude range, different values of $s$ are obtained, as can be seen in table 1 .

The variations of $s$ with time as seen in figure 1 (dotted line) are probably due to changes in the radiant distribution and the mass distribution. Known streams do not contribute substantially to the observed rates, since they are observed only for short period of time, if at all. However, unknown showers might be responsible for this scatter.

\section{ACKNOWLEDGMENTS}

I wish to thank M. Schaffner and R. B. Southworth for stimulating discussions and C. S. Nilsson for lending me one of his computer programs. This work was done under NASA contract NSR 09-015-033.

\section{REFERENCES}

Elford, W. G., AND Hawkins, G. S., 1964. Meteor echo rates and the flux of sporadic meteors, Harvard Radio Meteor Project Res. Rept. No. 9.

Hawkins, G. S., AND Upton, E. K. L., 1958. The influx rate of meteors in the Earth's atmosphere, Astrophys. $J$., 128, 727-735.

KAISER, T. R., 1961. The determination of the incident flux of radio meteors. II. Sporadic meteors, Monthly Notices Roy. Astron. Soc., 123, 265-271.

KRESÁKOVA, M., AND KRESÁK, L̆., 1955. On the activity of telescopic meteors and some related problems, Contrib. Astron. Obs. Skalnaté Pleso, 1, 40-77.

MCIntosh, B. A., AND Š̆IMEK, M., 1969. Mass distribution of meteoroids as determined by radar observations of underdense meteor trails, Can. J. Phys., 47, 7-22.

ÖPIK, E., 1958. Statistical results from the Arizona expedition for the study of meteors, Contrib. Armagh Obs., No. 26, 1-82.

SimeK, M., AND MCINTosh, B. A., 1968. Meteor mass distribution from underdense-trail echoes, in Physics and Dynamics of Meteors, edited by $\breve{L}$. Kresák and́ P. M. Millman, D. Reidel Publ. Co., Dordrecht, Holland, 362-372.

SouthwoRTh, R. B., Recombination in radar meteors, this volume.

Weiss, A. A., 1957. The distribution of the orbits of sporadic meteors, Australian J. Phys., 10, 77-102. 